\title{
Exotic Collatz cycles
}

by

\section{John L. Simons (Groningen)}

1. Introduction. The generalized Collatz problem (also called the $p x+q$ problem with $p$ an odd prime and $q$ odd) is defined by a sequence of natural numbers, generated conditionally by $x \mapsto x / 2$ if $x$ is even and by $x \mapsto(p x+q) / 2$ if $x$ is odd. We restrict ourselves to the case where $p \geq 5$ (prime), $q$ odd and $\operatorname{GCD}(p, q)=1$. For each factor $c>1$ of $q$, either none or all of the numbers $x_{i}$ in each trajectory (including a hypothetical cycle) must satisfy $\operatorname{GCD}\left(x_{i}, q\right)=c$. Because each cycle of the $p x+q$ problem with $\operatorname{GCD}\left(x_{i}, q\right)=c$ corresponds with a cycle of the $p x+q / c$ problem, we call cycles with $\operatorname{GCD}\left(x_{i}, q\right)=1$ primitive and any other cycle non-primitive. An $m$-cycle of the $p x+q$ problem has $m$ local minima $x_{i}$. We call an $m$-cycle $(m \geq 2)$ trivial if it is a multiple of an $m^{*}$-cycle with $m^{*}<m$. In a non-trivial $m$-cycle we have $x_{i} \neq x_{j}$ if $i \neq j$ and we assume in what follows that an $m$-cycle is non-trivial unless explicitly stated otherwise.

Simons [5] has proved that in an $m$-cycle $(p-2) x_{i}=a_{i} 2^{k_{i}}-q$ and consequently that a necessary and sufficient condition for the existence of an $m$-cycle is the existence of a solution $\left(a_{i}, k_{i}, l_{i}\right)$ of the diophantine system of equations

$$
\left(\begin{array}{cccc}
-p^{k_{0}} & 2^{k_{1}+l_{0}} & & \\
& -p^{k_{1}} & 2^{k_{2}+l_{1}} & \\
& & \ddots & \\
2^{k_{0}+l_{m-1}} & & & -p^{k_{m-1}}
\end{array}\right)\left(\begin{array}{c}
a_{0} \\
a_{1} \\
\vdots \\
a_{m-1}
\end{array}\right)=\left(\begin{array}{c}
q\left(2^{l_{0}}-1\right) \\
q\left(2^{l_{1}}-1\right) \\
\vdots \\
q\left(2^{l_{m-1}}-1\right)
\end{array}\right) .
$$

He further derives the following two existence conditions for 1-cycles:

LEMma 1. A necessary and sufficient condition for the existence of a 1 -cycle for the $p x+q$ problem is the existence of positive integers $k, l$ and $r$

2000 Mathematics Subject Classification: 11B83, 11J86.

Key words and phrases: generalized Collatz problem, cycle existence. 
(odd) such that $2^{k+l}-p^{k}=q \cdot r$ and the existence of an odd integer $x_{0}$ such that

$$
x_{0}=\frac{p^{k}-2^{k}}{(p-2) r} .
$$

Corollary 2. If, and only if,

$$
x_{0}=\frac{\left(p^{k}-2^{k}\right) q}{(p-2)\left(2^{k+l}-p^{k}\right)} \quad \text { and } \quad \operatorname{GCD}\left(x_{0}, q\right)=1
$$

then there exists a primitive 1-cycle with minimal element $x_{0}$.

Let $C(m, p, q)$ be the number of primitive $m$-cycles of the $p x+q$ problem and let $C(p, q)=\sum_{m} C(m, p, q)$. Let $B(m, p, q)$ be the number of primitive and non-primitive $m$-cycles of the $p x+q$ problem and let $B(p, q)=$ $\sum_{m} B(m, p, q)$. Let $S(p)$ be the set of primes $q$ such that

$$
2^{k+l}-p^{k}=q
$$

has a solution with $k \geq 2$. In this paper we derive the following results:

- For each $p$, there exist infinitely many $p x+q$ problems with $C(1, p, q)$ $\geq 1$.

- For each $p$ and $d>0$, there exist infinitely many $p x+q$ problems with $C(p, q)>d$.

- Let $p$ be fixed. If $S(p)$ is an infinite set (conjectured, no proof) then for each $d>0$ there exist infinitely many $p x+q$ problems ( $q$ prime) with $C(p, q)>d$.

Matthews [4] conjectured that (i) if $p=3$ then all trajectories end in a cycle, (ii) if $p \geq 5$ then almost all trajectories are divergent, (iii) $B(p, q)<\infty$. Lagarias [3] conjectured (i) $C(3, q) \geq 1$, (ii) $C(3, q)<\infty$ and proved that occasionally $C(3, q)$ takes large values. Brox [2] and Simons \& de Weger [6] proved $C(m, 3,1)<\infty$. Simons [5] proved $C(m, p, q)<\infty$. Belaga \& Mignotte [1] numerically showed that there exist $3 x+q$ problems with many primitive cycles. Our results formally agree with Matthews' second and third conjecture, however $p x+q$ problems with arbitrarily many cycles can be seen as exotic exceptions to the empirical divergent behavior of $p x+q$ problems $(p \geq 5)$.

2. Generalized Collatz sequences with $q$ odd. From Lemma 1 it follows directly that for each prime $p$ there exist infinitely many $q$-values for which the $p x+q$ problem has a 1-cycle. Simply set $q=2^{k+l}-p^{k}$ for each pair $(k, l)$. Then Lemma 1 applies with $r=1$. Consequently, the $p x+q$ problem has a 1-cycle with minimal element $x_{0}=\left(p^{k}-2^{k}\right) /(p-2)$. For each $p$ we can construct a value for $q$ such that the $p x+q$ problem has a primitive 1-cycle. 
Lemma 3. Let $p \geq 5$ be a prime. For each $k \geq 2$ and $l>\left(\log _{2} p-1\right) k>k$, let

$$
u=\frac{p^{k}-2^{k}}{p-2} \quad \text { and } \quad v=2^{k+l}-p^{k} .
$$

The $p x+q$ problem with $q=v / \operatorname{GCD}(u, v)$ has a primitive 1 -cycle.

Proof. Set $x_{0}=u / \operatorname{GCD}(u, v)$. Then

$$
x_{0}=\frac{u}{\operatorname{GCD}(u, v)}=\frac{u \cdot q}{v}=\frac{\left(p^{k}-2^{k}\right) q}{(p-2)\left(2^{k+l}-p^{k}\right)} .
$$

Further, we have

$$
\operatorname{GCD}\left(x_{0}, q\right)=\operatorname{GCD}\left(\frac{u}{\operatorname{GCD}(u, v)}, \frac{v}{\operatorname{GCD}(u, v)}\right)=1 .
$$

We now distinguish two cases:

1. $q=1$. Then $2^{k+l}-p^{k}=v=q \cdot v$ and $x_{0}=\left(p^{k}-2^{k}\right) /(p-2) v$. Hence Lemma 1 applies.

2. $q \geq 3$. Because $\operatorname{GCD}\left(x_{0}, q\right)=1$, Corollary 2 applies.

In both cases the $p x+q$ problem has a primitive 1-cycle.

Lemma 3 generates for fixed $p$ infinitely many values $q=v / \operatorname{GCD}(u, v)$ for which the $p x+q$ problem has a primitive 1-cycle. These $q$-values are not necessarily different. However, each new pair $(k, l)$ generates a new pair $(u, v)$. Now either the $p x+v$ problem has a primitive 1-cycle with $k$ odd and $l$ even elements or the $p x+q$ with $q=v / \operatorname{GCD}(u, v)$ problem has such a primitive 1-cycle. Because $C(1, p, v)<\infty$ and $C(1, p, q)<\infty$ the infinite sequence of pairs $\{k, l\}$ must generate an infinite sequence either of $p x+v$ problems with a primitive 1-cycle and/or an infinite sequence of $p x+q$ problems with a primitive 1-cycle. Hence we have

COROllary 4. For each $p$ there are infinitely many q-values for which the $p x+q$ problem has a primitive 1-cycle.

Consider as an example the case $p=5$ :

- For $k=2$ and $l=3$ we have $u=v=7$, hence $x_{0}=q=1$. The $5 x+1$ problem has the primitive 1-cycle $(1,3,8,4,2)$.

- For $k=2$ and $l=4$ we have $u=7$ and $v=39$, hence $x_{0}=7$ and $q=$ 39 . The $5 x+39$ problem has the primitive 1 -cycle $(7,37,112,56,28,14)$.

- For $k=2$ and $l=5$ we have $u=7$ and $v=103$, hence $x_{0}=7$ and $q=103$. The $5 x+103$ problem has the primitive 1 -cycle $(7,69$, $224,112,56,28,14)$.

- For $k=3$ and $l=4$ we have $u=39$ and $v=3$, hence $x_{0}=13$ and $q=1$. The $5 x+1$ problem has the primitive 1 -cycle $(13,33,83,208,104,52,26)$. 
Note that $q$ can be 1 , prime or composite. Because the $5 x+1$ problem has exactly two 1-cycles [5], for no other pair $(k, l)$ can reduction to the $5 x+1$ problem occur.

If for the $p x+q$ problem a 1-cycle exists with $k \geq 2$, then $m$-cycles with $m \geq 2$ also exist. This follows from the general solution of the system (1):

$$
\begin{aligned}
\Delta a_{i}= & q \cdot\left[p^{k_{i+1}+k_{i+2}+\cdots+k_{i-1}}\left(2^{l_{i}}-1\right)+2^{k_{i+1}+l_{i}} p^{k_{i+2}+\cdots+k_{i-1}}\left(2^{l_{i+1}}-1\right)\right. \\
& \left.+\cdots+2^{k_{i+1}+l_{i}+k_{i+2}+l_{i+1}+\cdots+k_{i-1}+l_{i-2}}\left(2^{l_{i-1}}-1\right)\right] .
\end{aligned}
$$

If $\Delta=2^{k+l}-p^{k}=q$ then each choice for $k_{i}$ and $l_{i}$ results in an integral $a_{i}$. Given $\sum_{i=0}^{m-1} k_{i}=k \geq 2$ and $\sum_{i=0}^{m-1} l_{i}=l \geq 3$ and any choice for $k_{i}$, we can choose (cyclic) different values for $l_{i}$ which result in a new $m$-cycle.

- The $5 x+7$ problem has the non-primitive 1 -cycle $(7,21,56,28,14)$ with $k=2$ and $l=3$. There exists one 2 -cycle: $\left(k_{0}=k_{1}=1, l_{0}=1, l_{1}=2\right)$ resulting in $(9,26,13,36,18)$, which is primitive.

- The $5 x+39$ problem has the primitive 1 -cycle $(7,37,112,56,28,14)$ with $k=2$ and $l=4$. There exist two 2-cycles: $\left(k_{0}=k_{1}=1, l_{0}=1\right.$, $\left.l_{1}=3\right)$ resulting in $(9,42,21,72,36,18)$, which is non-primitive, and $\left(k_{0}=k_{1}=1, l_{0}=l_{1}=2\right)$ resulting in $(13,52,26,13,52,26)$, which is non-primitive and trivial.

- The $5 x+103$ problem has the primitive 1 -cycle $(7,69,224,112,56$, $28,14)$ with $k=2$ and $l=5$. There exist two 2-cycles: $\left(k_{0}=k_{1}=1\right.$, $\left.l_{0}=1, l_{1}=4\right)$ resulting in $(9,74,37,144,72,36,18)$ and $\left(k_{0}=k_{1}=1\right.$, $\left.l_{0}=2, l_{1}=3\right)$ resulting in $(13,84,42,21,104,52,26)$. Both cycles are primitive.

- The $5 x+3$ problem has the non-primitive 1 -cycle $(39,99,249,624,312$, $156,78)$ with $k=3$ and $l=4$. There exist three 2-cycles: $\left(k_{0}=1\right.$, $\left.k_{1}=2, l_{0}=2, l_{1}=2\right)$ resulting in $(51,129,324,162,81,204,102)$, which is non-primitive, and $\left(k_{0}=1, k_{1}=2, l_{0}=1, l_{1}=3\right)$ resulting in $(43,109,274,137,344,172,86)$ and $\left(k_{0}=1, k_{1}=2, l_{0}=3, l_{1}=1\right)$ resulting in $(53,134,67,169,424,212,106)$, which are both primitive. There exists one 3-cycle: $\left(k_{0}=k_{1}=k_{2}=1, l_{0}=l_{1}=1, l_{2}=2\right)$ resulting in $(61,154,77,194,97,244,122)$, which is primitive.

We denote by $B^{*}(m, p, q)$ (resp. $\left.C^{*}(m, p, q)\right)$ the number of $m$-cycles (resp. primitive $m$-cycles) generated by partitioning from a 1-cycle. Clearly, $B(m, p, q) \geq B^{*}(m, p, q)$ etc. For special $p x+q$ problems, $B(m, p, q), C(2, p, q)$ and consequently $C(p, q)$ can be arbitrarily large.

Lemma 5. For each prime $p \geq 5, m$ and $d>0$ there exist infinitely many $q=2^{k+l}-p^{k}$ for which $B(m, p, q)>d$.

Proof. For fixed $p, k, l, m$, a (cyclic) new pair of partitions

$$
\left[\left(k_{0}, k_{1}, \ldots, k_{m-1}\right),\left(l_{0}, l_{1}, \ldots, l_{m-1}\right)\right]
$$


generates a new $m$-cycle. Note that $q$ follows from $p, k, l$. For fixed $p$ and $m$, $B^{*}(m, p, q)$ is an increasing function of $k$ and $l$. So for any $p, m$ and $d>0$ we can choose $k^{*}, l^{*}$ such that if $k>k^{*}$ and $l>l^{*}$ then $B^{*}(m, p, q)>d$. Consequently, $B(m, p, q) \geq B^{*}(m, p, q)>d$.

Lemma 6. For each prime $p \geq 5$ and $d>0$ there exist infinitely many $q=2^{k+l}-p^{k}$ for which $C(p, q) \geq C(2, p, q)>d$.

Proof. For each $d>0$ we can choose $k, l$ (hence $q$ ) such that $\operatorname{GCD}(k, l)$ $=1$ and $B^{*}(2, p, q)>2 d$. Let $x_{0}(j)$ be a local minimal element of the $j$ th 2 -cycle. From the matrix system (1) it follows for $k_{0}=k-1, k_{1}=1, l_{0}=j$, $l_{1}=l-j$ that

$$
a_{0}(j)=(p-2) 2^{j}+\left(2^{l+1}-p\right) .
$$

Using $(p-2) x_{0}=a_{0} 2^{k_{0}}-q$ we find

$$
x_{0}(j)=\frac{p^{k}-2^{k}}{p-2}+\left(2^{j}-1\right) \cdot 2^{k-1} .
$$

We observe that $x_{0}(0)$ is the global minimum of the 1-cycle, hence $x_{0}(j+1)$ $-x_{0}(j)=2^{j+k}$ for all $j$, from which it follows that if the $j$ th 2 -cycle is non-primitive, then the $(j-1)$ th and the $(j+1)$ th 2 -cycle is primitive. Such a primitive 2-cycle is non-trivial because $\operatorname{GCD}(k, l)=1$. As a consequence we find $C(p, q) \geq C(2, p, q) \geq C^{*}(2, p, q) \geq \frac{1}{2} B^{*}(2, p, q)>d$.

For $m \geq 3$ we cannot prove that $C(m, p, q)$ is arbitrarily large. For fixed $p, m, d$ and each $d^{*}>0$ we can choose $q$ such that $B(m, p, q) \geq B^{*}(m, p, q)>d^{*}$. Now suppose $C(m, p, q) \leq d \leq d^{*}$. Then for this pair $(p, q)$ the number of non-primitive $m$-cycles is $B(m, p, q)-C(m, p, q)>d^{*}-d \geq 0$. Each non-primitive $m$-cycle reduces to a different primitive $m$-cycle for a $p x+r$ problem with $r \mid q$. Now $C(m, p, r)<\infty$ and consequently $B^{*}(m, p, q) \leq$ $B(m, p, q)=\sum_{r} C(m, p, r)<\infty$. This upper bound for $B^{*}(m, p, q)$ could conflict with the lower bound $d^{*}$ if the number of different factors of $q$ is small enough. In such cases the assumption $C(m, p, q) \leq d \leq d^{*}$ cannot be true, so $C(m, p, q)$ is arbitrarily large. However if the number of different factors of $q$ is sufficiently large, the contradiction of the lower and upper bound of $C(m, p, q)$ cannot be reached.

3. Generalized Collatz sequences with $q$ an odd prime. Recall that $S(p)$ is the set of primes $q$ such that $2^{k+l}-p^{k}=q$ has a solution with $k \geq 2$, thereby excluding the trivial 1 -cycle $\left(1,2^{l-1}, \ldots, 2\right)$ for $p+q=2^{l}$. Such $p x+q$ problems form an interesting subset because of the empirical exceptionality of non-primitive cycles, i.e. $B(m, p, q) \simeq C(m, p, q)$. Also, for these $p x+q$ problems, $C(2, p, q)$ and consequently $C(p, q)$ can be arbitrarily large. 
Lemma 7. For fixed $p$, consider the set of $p x+q$ problems with $q \in S(p)$. If $S(p)$ is an infinite set, then for each $d>0$ there are infinitely many $p x+q$ problems with $C(2, p, q)>d$.

Proof. Let $q \in S(p)$ satisfy $B(2, p, q) \geq B^{*}(2, p, q) \simeq(k-1)(l-1) / 2$ $>2 d$. Consider the set of 2-cycles generated by partitioning from the 1cycle with $k$ odd and $l$ even elements, starting with $x_{0}=\left(p^{k}-2^{k}\right) /(p-2)$. Similarly to the proof of Lemma 6 we have $C^{*}(2, p, q) \geq \frac{1}{2} B^{*}(2, p, q)$. Consequently, $C(2, p, q) \geq C^{*}(2, p, q) \geq \frac{1}{2} B^{*}(2, p, q)>d$. Since $S(p)$ is an infinite set, for infinitely many $k^{*}>k$ and $l^{*}>l$ another $q^{*}$ (prime) results for which the same reasoning applies.

Note that $C(2, p, 1)<\infty$ and only exceptionally (empirical fact, no proof) $C(2, p, 1)>0$. For a generalization of the proof of Lemma 7 to $C(m, p, q)>d$, it is required that for every $k>1$ there exist infinitely many primes $q \in S(p)$. Since $C(p, q) \geq C(2, p, q)$ we have without this requirement:

COROllary 8. For fixed $p$, consider the set of $p x+q$ problems with $q \in S(p)$. If $S(p)$ is an infinite set, then for each $d>0$ there are infinitely many $p x+q$ problems with $C(p, q)>d$.

Trivial $m$-cycles can exist by definition, but they need not be generated by partitioning if $q$ is prime.

Lemma 9. If $2^{k+l}-p^{k}=q$ with $p, q$ prime and $k \geq 2$, for infinitely many $p$ all the $m$-cycles of the $p x+q$ problem, generated by partitions from a 1-cycle, are non-trivial.

Proof. For the existence of a trivial $m$-cycle we must have $\operatorname{GCD}(k, l)=$ $c>1$. Then $k=c \cdot v$ and $l=c \cdot w$ and $q=\left[2^{v+w}\right]^{c}-\left[p^{v}\right]^{c}=\left[2^{v+w}-p^{v}\right]$ $\cdot\left[2^{(v+w)(c-1)}+\cdots+p^{v(c-1)}\right]$. Now $q$ is prime and the second factor is $>p+2$, so $2^{v+w}-p^{v}=1$ hence $v=1$ and $p=2^{w+1}-1$. Consequently, if $p$ is not a Mersenne prime, then all generated $m$-cycles are non-trivial. If $p=2^{w+1}-1$ and $q=\sum_{j=0}^{c-1}\left(\begin{array}{c}c-1 \\ j\end{array}\right) 2^{(v+w)(c-1-j)} p^{v j}$ are both prime, then a non-trivial nonprimitive cycle with length $v+w$ exists.

As a consequence of Corollary 8 and Lemma 9 we have

Corollary 10. Consider the $p x+q$ problem with $p \geq 5$ prime and $q \in S(p)$. If $S(p)$ is an infinite set, then for each $p$ and $d>0$ there are infinitely many $p x+q$ problems with $C(p, q)>d$.

Simons [5] gives for $p<100$ the smallest $q \in S(p)$. They are shown in Table 1.

Exotic candidates are $p x+q$ problems with large values for $k$ and $l$. For example, the $23 x+4217$ problem with $k=3$ and $l=11$ has 26 primitive 
cycles, and the $97 x+32641759$ problem with $k=3$ and $l=22$ has 92 primitive cycles.

Table 1. $p x+q$ problem ( $q$ minimal) with primitive cycles

\begin{tabular}{rr|cc|cr}
\hline \multicolumn{1}{c}{$p$} & \multicolumn{1}{c|}{$q$} & $p$ & \multicolumn{1}{c|}{$q$} & $p$ & \multicolumn{1}{c}{$q$} \\
\hline 5 & 3 & 31 & 1087 & 67 & 28279 \\
7 & 79 & 37 & 6823 & 71 & 126031 \\
11 & 7 & 41 & 367 & 73 & 125743 \\
13 & 1879 & 43 & 199 & 79 & 1951 \\
17 & 223 & 47 & 30559 & 83 & 1303 \\
19 & 151 & 53 & 29959 & 89 & 271 \\
23 & 4217 & 59 & 29287 & 97 & 32641759 \\
29 & 7351 & 61 & 520567 & & \\
\hline
\end{tabular}

We computed $C(p, q)$ for $p=5,7$ and $q \in S(p)\left(q<10^{6}\right)$. These are presented in Table 2.

Table 2. $C(5, q)$ and $C(7, q)$ as a function of $k$ and $l$

\begin{tabular}{rccc|rccc}
\hline \multicolumn{1}{c}{$q$} & $k$ & $l$ & $C(5, q) \geq$ & $q$ & $k$ & $l$ & $C(7, q) \geq$ \\
\hline 3 & 3 & 4 & 3 & 79 & 2 & 5 & 3 \\
7 & 2 & 3 & 3 & 463 & 2 & 7 & 5 \\
103 & 2 & 5 & 3 & 1999 & 2 & 9 & 5 \\
131 & 3 & 5 & 9 & 5791 & 4 & 9 & 62 \\
487 & 2 & 7 & 4 & 30367 & 4 & 11 & 95 \\
971 & 5 & 7 & 66 & 32719 & 2 & 13 & 7 \\
1423 & 4 & 7 & 37 & 130729 & 3 & 14 & 40 \\
8167 & 2 & 11 & 6 & 131023 & 2 & 15 & 8 \\
13259 & 5 & 9 & 173 & 521887 & 4 & 15 & 206 \\
32143 & 4 & 11 & 95 & & & & \\
130447 & 4 & 13 & 140 & & & & \\
259019 & 5 & 13 & 489 & & & & \\
\hline
\end{tabular}

Notice that $B^{*}(p, q)$ is an increasing function of $k$ and $l$. These tables confirm that $C(p, q) \simeq B(p, q) \geq B^{*}(p, q)$ is an increasing function of $k$ and $l$.

4. Remarks. 1. The infinity of $S(p)$ is a conjecture (as the Mersenne conjecture). We found that $3,7,103,131,487,971,1423,8167,13259,32143$, 130447, 259019, 1706527, 4191179, 16699091, 16774091, 18280739, 33163807, $\ldots \in S(5)$. This $S(p)$ conjecture is milder than the Mersenne conjecture because $k \geq 2$ instead of $k=0$ is required for the primality of $q$. 
2. If $q \in S(p)$ and $C(1, p, q)=1$, then $B^{*}(p, q)$ can easily be calculated. Let $v(a, b)$ be the number of (cyclic) different and $w(a, b)$ be the total number of $b$-partitions of $a$. For fixed $k$ and $l$ (note that $l>k$ ) there are: one 1-cycle, $v(k, 2) \cdot w(l, 2) 2$-cycles etc. We checked that in many cases (not always) this lower bound equals the empirically found number of primitive cycles for $p=5,7$. Exceptions are the $5 x+7$ problem with no primitive 1-cycle and the $3 x+463$ problem with an extra 3 -cycle.

3 . For the $p x+q$ problem, the minimal elements in found cycles of equal length show a regular pattern. Let $x_{0}(m, j)$ be the minimal element of the $j$ th primitive $m$-cycle. From the matrix system (1) it follows for $m=2, k_{0}=$ $k-1, l_{0}=j$ that

$$
x_{0}(2, j)=\frac{p^{k}-2^{k}}{p-2}+\left(2^{j}-1\right) \cdot 2^{k-1} .
$$

A similar expression can be found for $x_{1}(2, j)$. For small $j$ we have $x_{0}<x_{1}$ and consequently successive minima of 2 -cycles for $j=1, \ldots, l-1$ differ by successive powers of 2 . Because the minimal element is $\min \left(x_{0}, x_{1}\right)$ this regular pattern can be disturbed for larger $j$.

4. For the $23 x+4217$ problem all cycles have length 14 with $k=3$ and $l=11$. We found next to $x_{0}(1,1)=579$ the sequence of minima: $x_{0}(2, j)=583,591,607,639,703,831,1087$ for 2 -cycles. The next elements of this sequence: $1599,2623,4671$, appear to be local minima in a 2-cycle, while the global minima are $929,729,629$. They form the beginning of a similar sequence for the global minima of 3 -cycles. So next to $x_{0}(2,9)=929$ we found $x_{0}(3, j)=961,1025,1152,1409$ for 3 -cycles etc.

5 . For the $7 x+521887$ problem, $k=4$ and $l=15$. From partitioning we found 204 cycles $(m \leq 4)$ with length 19 and with $x_{0}(m, j)$ in a regular pattern. We found two extra cycles $(m=5,6)$ with length 38 and with a minimal element smaller than $x_{0}(1,1)$.

6 . In this paper we have proved that $C(p, q)$ can be arbitrarily large. In view of the regular behavior of the minimal $x_{i}$ over the cycles we did not apply transcendence theory (as in [5]) to exclude cycles with large cycle length. This leaves theoretically open that $C(5, q)$ and $C(7, q)$ are greater than the numbers indicated in Table 2.

\section{References}

[1] E. G. Belaga and M. Mignotte, Cyclic structure of dynamical systems associated with the $3 x+d$ extensions of Collatz problem, http://www-irma.u-strasbg.fr/irma/publications/2000/00017.shtml.

[2] T. Brox, Collatz cycles with few descents, Acta Arith. 92 (2000), 181-188.

[3] J. C. Lagarias, The set of rational cycles for the $3 x+1$ problem, ibid. 56 (1990), $33-53$. 
[4] K. R. Matthews, The generalized 3x+1 mapping, http://www.numbertheory.org/pdfs/ survey.pdf.

[5] J. L. Simons, On the (non-)existence of $m$-cycles for generalized Syracuse sequences, Acta Arith. 131 (2008), 217-254.

[6] J. L. Simons and B. M. M. de Weger, Theoretical and computational bounds for $m$-cycles of the $3 n+1$-problem, ibid. 117 (2005), 51-70; see for latest results http:// www.win.tue.nl/ ${ }^{\sim}$ bdeweger/research.html.

University of Groningen

P.O. Box 800

9700 AV Groningen, The Netherlands

E-mail: j.l.simons@rug.nl

Received on 1.3.2007

and in revised form on 15.5.2008 